than females to disorders of the valves arising from strains. This may be inferred from the history of these cases; of the 24 males, 12 had no rheumatic history, 5 had had rheumatic pains, and 7 had had acute rheumatism; while of the 8 females, one had no rheumatic history, 2 had had rheumatic pains, and 5 had had rheumatic fever.

In contrast with the great frequency of valvular disease may be mentioned the fact that during the period under survey only three cases have presented themselves in which pericarditis was suspected, exclusive of cases in which pericardial adhesion might have been present. In one of these there was pericardial effusion along with endocardial mischief, and the case proved fatal a few days after being seen. In two others there was a suspicion of pericardial friction. It is to be supposed that, in the stages of friction and effusion, cases of pericarditis are too ill to undertake the exertion of a visit to the dispensary, those conditions generally coming on in the midst of an acute attack of rheumatism, and not developing out of the indefinite seizures that frequently give origin to valvular disease.

Symptoms.-I do not propose to discuss the well.known symptoms of cardiac disease, but I wish to direct attention to a few points in regard to these. The first is the absence of severe or even of any symptoms in many cases-a fact which was emphasised in that remarkable paper which Sir Andrew Clark read at the Brighton meeting of the British Medical Association." Many of my cases did not spontaneously complain of symptoms suggestive of cardiac disease, but in the great majority direct inquiry elicited a history of some cardiac symptoms or indications at a past date. Giving a much more liberal interpretation to what may be regarded as indications of cardiac affections than was done by Sir Andrew Clark, I find that in only 24 out of the 150 cases could absolutely no history of symptoms be obtained. In these and in many others the valvular mischief was discovered through examination of the heart being practically made a part of the routine to which patients are submitted.

Among the less common conditions suggestive of cardiac origin I would point out such as hæmaturia, of which there were two cases, and enlarged and painful spleen, of which there was one case, these being all regarded as of embolic origin. The only other embolic lesion noted was hemiplegia, of which there were seven cases, who were either suffering from that when seen or had a history of previous seizures. Besides these it may be noted that in one case melæna was a prominent symptom, and in three epistaxis. In one case an aortic lesion was associated with painful cramps affecting both legs and the left arm, which at first I was inclined to suppose might indicate embolism, but further observation tended to that idea being discarded. In three the disease was associated with fits, in one with purpura hæmorrhagica, and in one with herpes zoster.

Prognosis. - The question of prognosis resolves itself into a determination of the duration of the disease. Now, in the class of patients with whom we are dealing there is great difficulty in discovering when the cardiac disease originated, the great majority of them being quite ignorant of the fact that any such disease was present. Some were able to tell that the heart had been said to be affected at the date of some previous illness; but generally reliance had to be put upon the date of a rheumatic attack and the date of origin of symptoms as guides in determining this point, and it was found that the history was more clearly obtained in mitral lesions, and especially stenosis, than in any others, from the frequency with which hæmoptysis occ urred as an early symptom. In regard to this relation to rheumatism, however, it must be borne in mind that, perhaps not unfrequently, cardiac valvular lesion develops, not at the time of a rheumatic attack, but some time afterwards. It must also be premised that the cases were but rarely followed up to a termination, so that the actual duration could seldom be determined.

In 41 cases, or 27 per cent., the disease had in all probability lasted five years at least, and in 22 of these for ten years or more-viz., 7 aortic ( 6 double murmur, 1 pure regurgitation), and 15 mitral ( 6 stenosis, 8 incompetency, and 1 the combined lesion). These figures tend to confirm the teaching of Dr. Gairdner, that mitral lesion (perhaps especially mitral stenosis) is the form of valvular affection which is most compatible with prolongation of life.

(To be concluded.)
ON A CASE OF

\section{UNUNITED FRACTURE OF THE RADIUS SUCCESSFULLY TREATED BY THE GRAFTING OF RABBIT'S BONE.}

\section{BY A. F. MCGILL,}

PROFESSOR OF SURGERY IN THE YORKSHIRE COLLEGE, AND SURGEON TO THE LEEDS INHIRMARY.

THE treatment of ununited fracture of one of the bones of the forearm has, as far as I know, always been unsatisfactory. The reason is not difficult to find. In performing the usual operation of resection, it is necessary to remove portions from the end of each fragment, and it is then impossible to get the two portions into apposition. If the radius is broken the ulna prevents apposition, and if the ulna, the radius. To overcome this difficulty it has been proposed to cut down on the unfractured bone and remove a piece corresponding in size to the gap left by the resection. The bones being then wired there seems to be no reason why union should not occur. I have treated one case in this way, but the result was unsatisfactory. When the case which is the subject of this paper came under my care, it occurred to me that it was advisable to follow the line of treatment first suggested by Dr. MacEwen in a case of destruction of the shaft of the humerus from acute necrosisviz., the building up of new bone by the transplantation of bone fragments. The fragments in my case were taken from a young growing rabbit. The following are the notes taken by Mr. Kellett :-

J. $\mathbf{N}$-, aged twenty, was admitted into the Leeds Infirmary, under the care of Mr. McGill, on March 9th, 1889, suffering from an ununited fracture of the right radius. In February, 1888, he fractured his right forearm; the fracture was compound, the wound being on the radial side. Through the wound the broken ends of the radius protruded. The ulna united perfectly, but the fragments of the radius would not join. Three months after the accident a surgeon exposed the ends of the bone refreshed them, and wired them together. The wound healed, but the fragments did not unite. On admission, the radius was found to be fractured at the junction of the lower with the middle third. Over this position is the scar of a perfectly healed wound about two inches and a half in length. The two fragments of the radius are absolutely ununited, the utility of the forearm is much impaired, flexion of the fingers and adduction of the thumb being most imperfect. On March 19th, an Esmarch's bandage having been applied, Mr. McGill made an incision two or three inches in length in the situation of the old scar. Haring exposed the ends of the bone, he found that there was no sign of union, and that the two fragments were rounded at their extremities and covered by a thick membrane resembling periosteum. This was removed by means of a file, and bone tissue was thus bared. When this part of the operation was complete, an interval of about threequarters of an inch was left between the fragments. In the meantime a rabbit, six weeks old, had been killed, and small portions, one or two lines in length, had been chiselled from its femur. With these the interval between the fragments was filled up, thirteen pieces being inserted. The bones were not wired together. The skin wound was stitched up tightly with numerous catgut sutures. No drainage-tube was used. Firm pressure was applied by means of salicylic wool and bandages, and the forearm was placed on an anterior splint. The elastic tourniquet was not removed till the dressings were applied. On March 29th, the tenth day after the operation, the wound was dressed for the first time. There was no suppuration and very little discharge; the wound had healed, except a small superficial spot. The wool dressings were continued till April 16th, when there was slight irritation of the skin; they were consequently discontinued. The patient left the hospital on April 27th with the bone firmly uniced. In August he was slown at the meeting of the British Medical Association, when the injured arm was as useful as the other. Leeds.

BRISTOL Medical SCHOoL.-The annual dinner will take place at the Montague Hotel on Tuesday, Oct. 29th, F. P. Lansdown, Esq., in the chair. 